\title{
The approximate solutions of fractional gas dynamics equations by means of fractional natural decomposition method
}

Birol Ibis

National Defence University,Turkish Air Force Academy, Department of Basic Sciences, Istanbul,Turkey

Received: 16 December 2017, Accepted: 25 December 2017

Published online: 26 December 2017.

\begin{abstract}
In this work, a new practical method, which is named by the Fractional Natural Decomposition Method (FNDM), is proposed to obtain the approximate analytical solutions of fractional gas dynamics equations. The FNDM is a mixture of the Natural Transform Method and the Adomian Decomposition Method. In this method, the fractional derivatives are considered as Caputo sense and the nonlinear terms are determined by virtue of Adomian polynomials. Some test examples are given to demonstrate the efficiency and accuracy of the FNDM.
\end{abstract}

Keywords: Fractional gas dynamics equations, The Fractional Natural Decomposition Method (FNDM), Caputo Fractional Derivative, Adomian Polynomials.

\section{Introduction}

The concept of fractional calculus has been put forward in the study of derivatives and integrals of non-integer (arbitrary) order. Fractional calculus can be consider as the extension of the classical calculus theory and its attractiveness has increased considerably in the last four decades. Because the fractional calculus provides an adequate approach on modeling of the problems arise in science and engineering. The fractional differential equations (FDEs) have been widely used to model problems in electromagnetics, electric network, viscoelasticity, fluid mechanics, control theory, electrochemistry, biological population models, optics, signals processing, dynamical systems, thermodynamics, chemical physics and many other physical processes [1,2,3,4,5]. Most FDEs do not have exact analytic solutions. Therefore there have been many research works in the literature focus on finding the analytic, approximately analytical or numerical solutions of FDEs.

Gas dynamics equations are the equations which base on physical laws such as conservation of mass conservation of energy, conservation of momentum, etc [6]. The fractional gas dynamics equations have been the focus of some research works in the literature. Recently, various analytical and numerical methods have been developed to obtain solutions of fractional gas dynamics equations $[7,8,9,10,11,12,13,14,15,16,17]$.

The aim of this paper is to determine the approximate analytical solution of the time-fractional gas dynamics equations of the form:

$$
\left\{\begin{array}{l}
D_{t}^{\alpha} v+v v_{x}-v(1-v)=f(x, t), 0<\alpha \leq 1, t>0 \\
v(x, 0)=h(x), x \in \mathbb{R} .
\end{array}\right.
$$

where $t$ is the time, $x$ is the spatial coordinate and $v(x, t)$ is the probability density function. 


\section{Preliminaries}

Here, some basic concepts and properties with regard to fractional calculus are given.

Definition 1. The Riemann-Liouville ( $R$-L) fractional integral of $f(t) \in C_{\mu}, \mu \geq-1$ is defined by:

$$
\begin{gathered}
J^{\alpha} f(t)=\frac{1}{\Gamma(\alpha)} \int_{0}^{t}(t-\xi)^{\alpha-1} f(\xi) d \xi,(\alpha>0), \\
J^{0} f(t)=f(t) .
\end{gathered}
$$

For $f(t) \in C_{\mu}, \mu \geq-1, \gamma>-1, \beta, \alpha \geq 0$, some useful properties of the R-L fractional integral operator $J^{\alpha}$ are given by the following expressions:

$$
\begin{gathered}
J^{\alpha} J^{\beta} f(t)=J^{\alpha+\beta} f(t), \\
J^{\alpha} J^{\beta} f(t)=J^{\beta} J^{\alpha} f(t), \\
J^{\alpha} t^{\gamma}=\frac{\Gamma(\gamma+1)}{\Gamma(\gamma+\alpha+1)} t^{\alpha+\gamma} .
\end{gathered}
$$

Definition 2. The Caputo derivative of fractional order of $f(t)$ is given by

$$
D^{\alpha} f(t)=J^{n-\alpha} D^{n} f(t)=\frac{1}{\Gamma(n-\alpha)} \int_{0}^{t}(t-\xi)^{n-\alpha-1} f^{(n)}(\xi) d \xi, n-1<\alpha \leq n, n \in N, t>0 .
$$

For $f \in C_{\mu}^{n}, \mu \geq-1$, the Caputo derivative operator $D^{\alpha}$ satisfies the following properties:

$$
D^{\alpha}(\lambda f(t)+g(t))=\lambda D^{\alpha} f(t)+D^{\alpha} g(t)
$$

and,

$$
D^{\alpha} t^{k}= \begin{cases}\frac{k !}{\Gamma(k-\alpha+1)} t^{k-\alpha}, & k \geq \alpha \\ 0, & k<\alpha\end{cases}
$$

Definition 3. The Mittag-Leffler function is identified by

$$
E_{\alpha}(t)=\sum_{k=0}^{\infty} \frac{t^{k}}{\Gamma(\alpha k+1)},(\alpha \in C, \operatorname{Re}(\alpha)>0)
$$

Lemma 1. If $f \in C_{\mu}^{n}, \mu \geq-1$ and $n \in \mathbb{N}, n-1<\alpha \leq n$, then

$$
\begin{gathered}
D^{\alpha} J^{\alpha} f(x)=f(x) \\
J^{\alpha} D^{\alpha} f(x)=f(x)-\sum_{k=0}^{n-1} f^{(k)}\left(0^{+}\right) \frac{x^{k}}{k !}, x>0 .
\end{gathered}
$$




\section{The natural transform}

The Natural transform (N-transform), which is an integral transform, is defined and studied its properties and applications [18]. Later, Belgacem and Silambarasan defined inverse of natural transform and studied some additional fundamental properties of its [19]. Recently, $\mathrm{N}$ - transform is used to find the solution of differential and integral equations [21,22,23, $24,25,26,27,28]$.

Definition 4. The $N$-transform of $f(t)$ for all $t \geq 0$, is defined as:

$$
\begin{aligned}
\mathbb{N}[f(t)] & =R(s, u)=\int_{0}^{\infty} e^{-s t} f(u t) d t=\frac{1}{u} \int_{0}^{\infty} e^{-\frac{s t}{u}} f(t) d t \\
& =\frac{1}{s} \int_{0}^{\infty} e^{-t} f\left(\frac{u t}{s}\right) d t ; \operatorname{Re}(s)>0, u \in\left(\tau_{1}, \tau_{2}\right)
\end{aligned}
$$

where $s$ is the frequency variable and $t, u$ are time variables, $f(t)$ is defined in the set $A=\left\{f(t)\left|\exists M, \tau_{1}, \tau_{2}>0,\right| f(t) \mid<\right.$ $M e^{|t|} / \tau_{j}$,if $\left.t \in(-1)^{j} \times[0, \infty)\right\}\left(M\right.$ is constant, $\tau_{1}$ and $\tau_{2}$ may be finite or infinite $)$.

Definition 5. The inverse $N$-transform is defined as:

$$
\mathbb{N}^{-1}[R(s, u)]=f(t)=\frac{1}{2 \pi i} \int_{c-i \infty}^{c+i \infty} e^{\frac{s t}{u}} R(s, u) d s
$$

The N-transform and the inverse N-transform are linear operators. Also, the N-transform have the following some properties [13, 19,20,22,23].

Theorem 1. The $N$-transform of the $n$-th order derivative of $f(t)$ with respect to $t$ is defined as:

$$
\mathbb{N}\left[f^{(n)}(t)\right]=\frac{s^{n}}{u^{n}} R(s, u)-\sum_{k=0}^{n} \frac{s^{n-k-1}}{u^{n-k}} f^{(k)}(0)
$$

Theorem 2. Let the $N$-transforms of $f(t)$ and $g(t)$ be $F(s, u)$ and $G(s, u)$ respectively, then the convolution of $f(t)$ and $g(t)$ is given by

$$
\mathbb{N}[(f * g)(t)]=u F(s, u) G(s, u)
$$

Theorem 3. If $f(t)=\delta(t)$, then the $N$-transform of $f(t)$ is:

$$
\mathbb{N}[\delta(t)]=\frac{1}{u}
$$

Theorem 4. If $f(t)=t^{\alpha}(\alpha>-1)$, then the $N$-transform of $f(t)$ is:

$$
\mathbb{N}[f(t)]=\mathbb{N}\left[t^{\alpha}\right]=\frac{\Gamma(\alpha+1) u^{\alpha}}{s^{\alpha+1}}
$$

Theorem 5. Let the $N$-transforms of $f(t)$ is $R(s, u)$, then $N$-transform of $t^{n} f(t)$ is: 


$$
\mathbb{N}\left[t^{n} f(t)\right]=\frac{u^{n}}{s^{n}} \frac{d^{n}}{d u^{n}} u^{n} R(s, u)
$$

Theorem 6. The $N$-transform of the $R$-L fractional integral of $f(t)$ is:

$$
\mathbb{N}\left[J^{\alpha} f(t)\right]=\frac{u^{\alpha}}{s^{\alpha}} \mathbb{N}[f(t)]
$$

Theorem 7. The N-transform of the Caputo fractional derivative of $f(t)$ is:

$$
\mathbb{N}\left[D^{\alpha} f(t)\right]=\frac{s^{\alpha}}{u^{\alpha}} \mathbb{N}[f(t)]-\sum_{k=0}^{n-1} \frac{s^{\alpha-k-1}}{u^{\alpha k}} D^{k} f(0) .
$$

\section{Analysis of FNDM}

In this section,the FNDM has been introduced widely. The following general class of the FDEs are considered:

$$
\left\{\begin{array}{l}
D_{t}^{\alpha} v(x, t)+L v(x, t)+N v(x, t)=f(x, t), t>0,0<\alpha \leq 1, \\
v(x, 0)=h(x), x \in \mathbb{R}
\end{array}\right.
$$

where $D_{t}^{\alpha}$ is the Caputo derivative, $N$ is nonlinear operator and $L$ is linear operator. If we implement the $\mathrm{N}$-transform to both sides of (22) and use the theorem 7, we get

$$
\begin{gathered}
\mathbb{N}\left[D_{t}^{\alpha} v(x, t)+L v(x, t)+N v(x, t)\right]=\mathbb{N}[f(x, t)] \\
\mathbb{N}[v(x, t)]=\frac{u^{\alpha}}{s^{\alpha}} \sum_{k=0}^{n-1} \frac{s^{\alpha-k-1}}{u^{\alpha-k}}\left[D_{t}^{k} v(x, t)\right]_{t=0}-\frac{u^{\alpha}}{s^{\alpha}} \mathbb{N}[L v(x, t)+N v(x, t)]+\frac{u^{\alpha}}{s^{\alpha}} \mathbb{N}[f(x, t)] \\
\mathbb{N}[v(x, t)]=\frac{1}{s} h(x)-\frac{u^{\alpha}}{s^{\alpha}} \mathbb{N}[L v(x, t)+N v(x, t)]+\frac{u^{\alpha}}{s^{\alpha}} \mathbb{N}[f(x, t)]
\end{gathered}
$$

After that, let us take the inverse N-transform on both sides of (25). So,

$$
v(x, t)=g(x, t)-\mathbb{N}^{-1}\left[\frac{u^{\alpha}}{s^{\alpha}} \mathbb{N}\left[L v_{x}(x, t)+N v(x, t)\right]\right]
$$

where

$$
g(x, t)=\mathbb{N}^{-1}\left[\frac{1}{s} h(x)+\frac{u^{\alpha}}{s^{\alpha}} \mathbb{N}[f(x, t)]\right]=h(x)+\mathbb{N}^{-1}\left[\frac{u^{\alpha}}{s^{\alpha}} \mathbb{N}[f(x, t)]\right]
$$

Let us consider that $v(x, t)=\sum_{n=0}^{\infty} v_{n}(x, t)$ is the solution and the nonlinear term is dissociated as $N v(x, t)=\sum_{n=0}^{\infty} A_{n}$ for some Adomian polynomials $A_{n}$. So, we can rewrite (26) as

$$
\sum_{n=0}^{\infty} v_{n}(x, t)=g(x, t)-\mathbb{N}^{-1}\left[\frac{u^{\alpha}}{s^{\alpha}} \mathbb{N}\left[L\left(\sum_{n=0}^{\infty} v_{n}(x, t)\right)+\sum_{n=0}^{\infty} A_{n}\right]\right]
$$


Matching both sides of (28) we have the following relation,

$$
\begin{aligned}
& v_{0}(x, t)=g(x, t), \\
& v_{1}(x, t)=-\mathbb{N}^{-1}\left[\frac{u^{\alpha}}{s^{\alpha}} \mathbb{N}\left[L v_{0}(x, t)+A_{0}\right]\right], \\
& v_{2}(x, t)=-\mathbb{N}^{-1}\left[\frac{u^{\alpha}}{s^{\alpha}} \mathbb{N}\left[L v_{1}(x, t)+A_{1}\right]\right],
\end{aligned}
$$

Thus, the following recursive relation is obtained:

$$
v_{n+1}(x, t)=-\mathbb{N}^{-1}\left[\frac{u^{\alpha}}{s^{\alpha}} \mathbb{N}\left[L v_{n}(x, t)+A_{n}\right]\right], n \geq 1 .
$$

So that the aproximate solution is given by

$$
v(x, t)=\sum_{n=0}^{\infty} v_{n}(x, t) .
$$

\section{Applications of the FNDM}

In this section, by using the present method, several fractional gas dynamics equations in form of (1) are solved to illustrate how the FNDM works and to show effectiveness and accuracy of the FNDM.

Example 1. Let us take the homogenous fractional gas dynamics equation $[13,17]$ as follows.

$$
\left\{\begin{array}{l}
D_{t}^{\alpha} v+v v_{x}-v(1-v)=0, t>0,0<\alpha \leq 1 \\
v(x, 0)=e^{-x}, x \in \mathbb{R}
\end{array}\right.
$$

Taking N-transform of (32), then using theorem 7 and the initial condition in (29), we have

$$
\begin{gathered}
\mathbb{N}\left[D_{t}^{\alpha} v\right]=\mathbb{N}\left[-v v_{x}+v-v^{2}\right] \\
\mathbb{N}[v]=\frac{1}{s} e^{-x}-\frac{u^{\alpha}}{s^{\alpha}} \mathbb{N}\left[v v_{x}-v+v^{2}\right]
\end{gathered}
$$

Taking the inverse N-transform of (34), we get:

$$
v(x, t)=e^{-x}-\mathbb{N}^{-1}\left[\frac{u^{\alpha}}{s^{\alpha}} \mathbb{N}\left[v v_{x}-v+v^{2}\right]\right]
$$

Now, we define $v(x, t)=\sum_{n=0}^{\infty} v_{n}(x, t)$ as the solution and the nonlinear terms $v v_{x}$ and $v^{2}$ are dissociated as

$$
v v_{x}=\sum_{n=0}^{\infty} A_{n}, v^{2}=\sum_{n=0}^{\infty} B_{n}
$$

where $A_{n}$ and $B_{n}$ are Adomian polynomials, then (35) will become:

$$
\sum_{n=0}^{\infty} v_{n}(x, t)=e^{-x}-\mathbb{N}^{-1}\left[\frac{u^{\alpha}}{s^{\alpha}} \mathbb{N}\left[\sum_{n=0}^{\infty} A_{n}-\sum_{n=0}^{\infty} v_{n}+\sum_{n=0}^{\infty} B_{n}\right]\right], n \geq 0
$$


Then matching both sides of (37), we could be able to calculate some of the terms of the series as follows:

$$
\begin{aligned}
& v_{0}(x, t)=e^{-x} \\
& v_{1}(x, t)=-\mathbb{N}^{-1}\left[\frac{u^{\alpha}}{s^{\alpha}} \mathbb{N}\left[A_{0}-v_{0}+B_{0}\right]\right]=-\mathbb{N}^{-1}\left[\frac{u^{\alpha}}{s^{\alpha}} \mathbb{N}\left[v_{0 x} v_{0}-v_{0}+v_{0}^{2}\right]\right]=e^{-x} \frac{t^{\alpha}}{\Gamma(\alpha+1)}, \\
& v_{2}(x, t)=-\mathbb{N}^{-1}\left[\frac{u^{\alpha}}{s^{\alpha}} \mathbb{N}\left[A_{1}-v_{1}+B_{1}\right]\right]=-\mathbb{N}^{-1}\left[\frac{u^{\alpha}}{s^{\alpha}} \mathbb{N}\left[v_{0 x} v_{1}+v_{0} v_{1 x}-v_{1}+2 v_{0} v_{1}\right]\right]=e^{-x} \frac{t^{2 \alpha}}{\Gamma(2 \alpha+1)}, \\
& v_{3}(x, t)=-\mathbb{N}^{-1}\left[\frac{u^{\alpha}}{s^{\alpha}} \mathbb{N}\left[A_{2}-v_{2}+B_{2}\right]\right]=-\mathbb{N}^{-1}\left[\frac{u^{\alpha}}{s^{\alpha}} \mathbb{N}\left[v_{0 x} v_{2}+v_{1 x} v_{1}+v_{2 x} v_{0}-v_{2}+2 v_{0} v_{1}+v_{1}^{2}\right]\right]=e^{-x} \frac{t^{3 \alpha}}{\Gamma(3 \alpha+1)},
\end{aligned}
$$

If we continue in this way, the following approximate solution is obtained

$$
\begin{aligned}
v(x, t) & =\sum_{n=0}^{\infty} v_{n}(x, t) \\
& =e^{-x}\left[1+\frac{t^{\alpha}}{\Gamma(\alpha+1)}+\frac{t^{2 \alpha}}{\Gamma(2 \alpha+1)}+\frac{t^{3 \alpha}}{\Gamma(3 \alpha+1)}+\ldots\right] \\
& =e^{-x} \sum_{k=0}^{\infty} \frac{t^{k \alpha}}{\Gamma(k \alpha+1)} \\
& =e^{-x} E_{\alpha}\left(t^{\alpha}\right)
\end{aligned}
$$

If we take $\alpha=1$, then $v(x, t)=e^{t-x}$ which is an exact solution of (32).

Example 2.Let us assume the homogenous fractional gas dynamics equation [13,17] given as:

$$
\left\{\begin{array}{l}
D_{t}^{\alpha} v+v v_{x}-v(1-v) \log a=0,0<\alpha \leq 1, a, t>0 \\
v(x, 0)=a^{-x}, x \in \mathbb{R}
\end{array}\right.
$$

Taking the N-transform of (40), then using theorem 7 and the initial condition in (40), we have

$$
\begin{gathered}
\mathbb{N}\left[D_{t}^{\alpha} v\right]=-\mathbb{N}\left[v v_{x}-\left(v-v^{2}\right) \log a\right] \\
\mathbb{N}[v]=\frac{1}{s} a^{-x}-\frac{u^{\alpha}}{s^{\alpha}} \mathbb{N}\left[v v_{x}\right]+\frac{u^{\alpha}}{s^{\alpha}} \cdot \log a \cdot \mathbb{N}\left[v-v^{2}\right]
\end{gathered}
$$

Taking the inverse N-transform of (42), we get:

$$
v(x, t)=a^{-x}-\mathbb{N}^{-1}\left[\frac{u^{\alpha}}{s^{\alpha}} \mathbb{N}\left[v v_{x}\right]\right]+\log a \cdot \mathbb{N}^{-1}\left[\frac{u^{\alpha}}{s^{\alpha}} \mathbb{N}\left[v-v^{2}\right]\right]
$$

Now, let define $v(x, t)=\sum_{n=0}^{\infty} v_{n}(x, t)$ as the solution and the nonlinear terms $v v_{x}$ and $v^{2}$ are dissociated as

$$
v v_{x}=\sum_{n=0}^{\infty} A_{n}, v^{2}=\sum_{n=0}^{\infty} B_{n}
$$

Here $A_{n}$ and $B_{n}$ are Adomian polynomials. Then (43) will become:

$$
\sum_{n=0}^{\infty} v_{n}(x, t)=a^{-x}-\mathbb{N}^{-1}\left[\frac{u^{\alpha}}{s^{\alpha}} \mathbb{N}\left[\sum_{n=0}^{\infty} A_{n}\right]\right]+\log a \cdot \mathbb{N}^{-1}\left[\frac{u^{\alpha}}{s^{\alpha}} \mathbb{N}\left[\sum_{n=0}^{\infty} v_{n}-\sum_{n=0}^{\infty} B_{n}\right]\right], n \geq
$$


Then matching both sides of (45), some of the terms of the series can be determined as follows:

$$
\begin{aligned}
v_{0}(x, t) & =a^{-x}, \\
v_{1}(x, t) & =-\mathbb{N}^{-1}\left[\frac{u^{\alpha}}{s^{\alpha}} \mathbb{N}\left[A_{0}\right]\right]+\log a \cdot \mathbb{N}^{-1}\left[\frac{u^{\alpha}}{s^{\alpha}} \mathbb{N}\left[v_{0}-B_{0}\right]\right] \\
& =-\mathbb{N}^{-1}\left[\frac{u^{\alpha}}{s^{\alpha}} \mathbb{N}\left[v_{0 x} v_{0}\right]\right]+\log a \cdot \mathbb{N}^{-1}\left[\frac{u^{\alpha}}{s^{\alpha}} \mathbb{N}\left[v_{0}-v_{0}^{2}\right]\right] \\
& =a^{-x} \frac{t^{\alpha} \log a}{\Gamma(\alpha+1)}, \\
v_{2}(x, t) & =-\mathbb{N}^{-1}\left[\frac{u^{\alpha}}{s^{\alpha}} \mathbb{N}\left[A_{1}\right]\right]+\log a \cdot \mathbb{N}^{-1}\left[\frac{u^{\alpha}}{s^{\alpha}} \mathbb{N}\left[v_{1}-B_{1}\right]\right] \\
& =-\mathbb{N}^{-1}\left[\frac{u^{\alpha}}{s^{\alpha}} \mathbb{N}\left[v_{0 x} v_{1}+v_{0} v_{1 x}\right]\right]+\log a \cdot \mathbb{N}^{-1}\left[\frac{u^{\alpha}}{s^{\alpha}} \mathbb{N}\left[v_{1}-2 v_{0} v_{1}\right]\right] \\
& =a^{-x} \frac{t^{2 \alpha} \log ^{2} a}{\Gamma(2 \alpha+1)}, \\
v_{3}(x, t) & =-\mathbb{N}^{-1}\left[\frac{u^{\alpha}}{s^{\alpha}} \mathbb{N}\left[A_{2}\right]\right]+\log a \cdot \mathbb{N}^{-1}\left[\frac{u^{\alpha}}{s^{\alpha}} \mathbb{N}\left[v_{2}-B_{2}\right]\right] \\
& =-\mathbb{N}^{-1}\left[\frac{u^{\alpha}}{s^{\alpha}} \mathbb{N}\left[v_{0 x} v_{2}+v_{1 x} v_{1}+v_{2 x} v_{0}\right]\right]+\log a \cdot \mathbb{N}^{-1}\left[\frac{u^{\alpha}}{s^{\alpha}} \mathbb{N}\left[v_{2}-2 v_{0} v_{1}+v_{1}^{2}\right]\right] \\
& =a^{-x} \frac{t^{3 \alpha} \log ^{3} a}{\Gamma(3 \alpha+1)} .
\end{aligned}
$$

We continue in this manner, we obtain the approximate solution given by

$$
\begin{aligned}
v(x, t) & =\sum_{n=0}^{\infty} v_{n}(x, t) \\
& =a^{-x}\left[1+\frac{t^{\alpha} \log a}{\Gamma(\alpha+1)}+\frac{t^{2 \alpha} \log ^{2} a}{\Gamma(2 \alpha+1)}+\frac{t^{3 \alpha} \log ^{3} a}{\Gamma(3 \alpha+1)}+\ldots\right] \\
& =a^{-x} \sum_{k=0}^{\infty} \frac{t^{k \alpha} \log ^{k} a}{\Gamma(k \alpha+1)} \\
& =a^{-x} E_{\alpha}\left(t^{\alpha} \log a\right) .
\end{aligned}
$$

If we take $\alpha=1$, then $v(x, t)=a^{-x+t}$ which is an exact solution of (40).

\section{Conclusion}

In this work, the FNDM is applied to determine the approximate solutions of some nonlinear fractional gas dynamics equations. It provides a series solution which converges rapidly and fluently to an exact or approximate solution. Some test examples are demonstrated to show the efficiently and high justice of the method. So, the FNDM can be further implemented to solve fractional differential and integral equations.

\section{Competing interests}

The authors declare that they have no competing interests. 


\section{Authors' contributions}

All authors have contributed to all parts of the article. All authors read and approved the final manuscript.

\section{References}

[1] K.B. Oldham and J. Spanier, Fractional Calculus: Theory and Applications, Differentiation and Integration to Arbitrary Order, New York: Academic Press, 1974.

[2] I. Podlubny, Fractional Differential Equations, an Introduction to Fractional Derivatives, Fractional Differential Equations, Some Methods of Their Solution and Some of Their Applications, Academic Press, 1999.

[3] A. A. Kilbas, H.M. Srivastava and J.J. Trujillo, Theory and Application of Fractional Differential Equations. North Holland Mathematics Studies, 204, 2006.

[4] F. Biagini F., Y. Hu , B. Oksendal and T. Zhang, Stochastic Calculus for Fractional Brownian Motion and Applications, SpringerVerlag, London, 2008.

[5] F. Mainardi, Fractional Calculus and Waves in Linear Viscoelasticity, An Introduction to Mathematical Models, Imperial College Press, London, 2010.

[6] J. L. Steger and R.F. Warming; Flux vector splitting of the inviscid gas dynamic equations with application to finite-difference methods, Journal of Computational Physics., 40, 263293, 1981. doi:10.1016/0021-9991(81)90210-2

[7] D. J. Evans and H. Bulut, A new approach to the gas dynamics equation: an application of the decomposition method, International Journal of Computer Mathematics, 79(7),817-822, 2002.

[8] H. Jafari, C. Chun, S. Seifi, and M. Saeidy, Analytical solution for nonlinear gas dynamic equation by homotopy analysis method, Applications and Applied Mathematics, 4(1), 149-154, 2009.

[9] S. Das, R. Kumar, Approximate analytical solutions of fractional gas Dynamics, Applied Mathematics and Computation, 217(24), 9905-9915, 2011.

[10] S. Kumar, H. Kocak and A. Yildirim, A Fractional Model of Gas Dynamics Equations and its Analytical Approximate Solution Using Laplace Transform. Zeitschrift fur Naturforschung A, 67(6-7), 389-396,2012.

[11] J. Singh, D. Kumar, A. Kilicman, Homotopy perturbation method for fractional gas dynamics equation using Sumudu transform, Abstract and Applied Analysis, vol. 2013, Article ID 934060, 8 pages, 2013. doi:10.1155/2013/934060

[12] H. Aminikhah and A. Jamalian, Numerical approximation for nonlinear gas dynamic equation, International Journal of Partial Differential Equations, vol. 2013, Article ID 846749, 7 pages, 2013.

[13] S. Kumar and M. Rashidi, New analytical method for gas dynamics equation arising in shock fronts, Computer Physics Communications, 185, 1947-1954,2014.

[14] A. Esen and O. Tasbozan, An approach to time fractional gas dynamics equation: quadratic B-spline Galerkin method, Applied Mathematicsand Computation, 261, 330-336,2015.

[15] O.S. Iyiola, On the solutions of nonlinear time fractional gas dynamics equations an analytical approach, International Journal of Pure and Applied Mathematics (IJPAM), 98(4), 491-502,2015.

[16] A. Esen, B. Karaagac and O. Tasbozan, Finite Difference Methods for Fractional Gas Dynamics Equation, Applied Mathematics and Information Sciences Letters, 4(1), 1-4, 2016.

[17] M. Tamsir and V.K. Srivastava, Revisiting the approximate analytical solution of fractional-order gas dynamics equation, Alexandria Engineering Journal, 55 (2), 867-874,2016.

[18] Z. H. Khan and W. A. Khan, N-transform properties and applications, NUST Journal of Engineering Sciences, 1, 127-133,2008.

[19] R. Silambarasn and F.B.M. Belgacem, Applications of the natural transform to Maxwell's quations , Progress In Electromagnetics Research Symposium Proceedings, Suzhou, China, Sept. 12-16, 2011.

[20] S.K.Q. Al-Omari, On the application of natural transforms, International Journal of Pure and Applied Mathematics 85(4), 729-744, 2013.

[21] M.S. Rawashdeh and S. Maitama, Solving nonlinear ordinary differential equations using the NDM, Journal of Applied Analysis and Computation, 5(1), 77-88, 2015.

[22] M. Omran, A. Kilicman and X. Zhang, Natural transform of fractional order and some properties, Cogent Mathematics, 3(1), 2016. 
[23] S. Maitama, A new analytical approach to linear and nonlinear partial differential equations Nonlinear Studies, 23(4), 675-684, 2016.

[24] M.S. Rawashdeh and H. Al-Jammal, New approximate solutions to fractional nonlinear systems of partial differential equations ung the FNDM, Advance Differential Equations, 2016(1), 235, 2016.

[25] S. Rida, A. Arafa, A. Abedl-Rady and H. Abdl-Rahaim, Fractional physical differential equations via natural transform, Chinese Journal of Physics, 55(4), 1569-1575, 2017.

[26] M. Rawashdeh and S. Maitama, Finding exact solutions of nonlinear PDEs using the natural decomposition method, Mathematical Methods in the Applied Sciences,40(1), 223-236, 2017.

[27] M.S. Rawashdeh, The fractional natural decomposition method: theories and applications, Mathematical Methods in the Applied Sciences,40(7), 2362-2376, 2017.

[28] F.B.M. Belgacem and R.Silambarasan, Theory of Natural Transform, Mathematics in Engineering, Science and Aerospace (MESA),3(1), 99-124, 2012. 